\title{
VIRUS
}

\section{Beiträge zur Sozialgeschichte der Medizin}

\author{
Band 13
}

Schwerpunkt:

Alternative und komplementäre Heilmethoden in der Neuzeit

\author{
Herausgegeben von
}

Elisabeth Lobenwein, Gerhard Ammerer und Alfred Stefan Weiß

für den Verein für Sozialgeschichte der Medizin

Leipzig: Leipziger Universitätsverlag, 2015

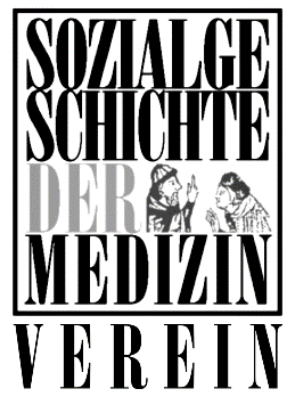




\section{„Alternative und komplementäre Heilmethoden in der Neuzeit"6 Eine kritische Annäherung an die Jahrestagung 2013 „Geschichte(n) von Gesundheit und Krankheit“" des Vereins für Sozialgeschichte der Medizin}

\section{English Title}

"Alternative and Complementary Medicine in the Modern Age." A Critical Approach to the Austrian Society for the Social History of Medicine's Annual Conference "Histories of Health and Illness".

\section{Summary}

This paper discusses the 2013 annual conference of the Austrian Society for the Social History of Medicine, choosing two different angles of approach. On the one hand the article gives a brief overview of the individual talks held at the conference dealing with alternative and complementary medicine from the early modern period to the present. On the other hand it tries to identify common motives and recurring concepts in the conference talks and papers published in this volume. Examples reach from magico-religious phenomena, natural medicine and the strong life reform movement of the fin de siècle to various agents of alternative medicine such as proponents of Cartesianism, Homeopathy or lay-healers. Similarities could be found in the evolution of alternative medical approaches, the recollection of antique dietetics, observed in many cases as well as the intriguing self-concept of many healers.

\section{Keywords}

Alternative medicine, complementary medicine, modern age, conference overview, critical analyses 


\section{Einleitung}

Im Jahr 1996 konstatierte Robert Jütte in seiner „Geschichte der Alternativen Medizin“ die „zunehmende Beliebtheit“ alternativer Heilmethoden in Europa. ${ }^{1}$ Diese Aussage lässt sich beinahe 20 Jahre nach dem Druck seines Standardwerkes keineswegs entkräften, erfreuen sich alternative und/oder komplementäre Zugänge zur Therapie kranker und zur Erhaltung gesunder Körper auch im neuen Jahrtausend äußerster Beliebtheit. Der Verein für Sozialgeschichte der Medizin griff den aktuellen Trend hin zur Alternativmedizin auf und widmete sich in seiner Jahrestagung 2013 dem Spannungsfeld alternativer Therapieformen und Methoden zur Gesunderhaltung, wie sie seit der Frühen Neuzeit dokumentiert und praktiziert wurden. Vom 8. bis zum 11. Mai 2013 wurden im Stadtarchiv Salzburg somit verschiedenste historische sowie aktuelle therapeutische Konzepte, Zugänge und Entwicklungen beleuchtet und deren Verankerung im breiten Feld der Alternativ- und/oder Komplementärmedizin diskutiert. Die Tagung orientierte sich an einem mehr oder weniger chronologischen Schema, welches die TeilnehmerInnen in mehreren Sektionen von der Frühen Neuzeit bis in die Gegenwart führte. Um die Kohärenz der folgenden Ausführungen - die mehr als eine kritische Zusammenschau, denn als ein einfacher Tagungsbericht verstanden werden sollen - zu erhöhen, wird diese Chronologie zugunsten eines thematischen Zugangs aufgebrochen.

Die einzelnen Tagungsbeiträge und Aufsätze des vorliegenden Bandes sind einerseits im Bereich der religiös-magischen Medizin, der Naturheilverfahren sowie der biodynamischen Heilweisen und fernöstlichen Medizin zu verorten, andererseits fokussieren die Beiträge mit ihrem Blick auf die Anbieter alternativer Praktiken auch die verstärkt um 1800 einsetzenden Professionalisierungs- und Hierachisierungsbestrebungen einer naturwissenschaftlich begründeten Medizin gegenüber nicht universitär approbierten Heilanbietern.

\section{Vom Wesen der Alternativmedizin}

Der Vortragsreigen wurde mit einem wegweisenden Beitrag von Heinz DOPSCH (1942-2014) (Salzburg) eröffnet, welcher anhand eines Fallbeispiels die zugrundeliegende Frage nach dem Wesen der Alternativmedizin und ihrer Akteure problematisierte. Dopsch widmete sich in seinen Ausführungen dem vermeintlichen Vater der Naturheilkunde, Theophrastus Bombastus von Hohenheim (1493-1541), allgemein bekannt unter dem Namen Paracelsus. Dessen tradierter Status als Wegbereiter einer alternativen Medizin und Bahnbrecher in verschiedenen anderen Bereichen der etablierten Medizin (Chirurgie, Anatomie, Balneologie, Psychiatrie, Arbeitsmedizin) sei zu hinterfragen, so der Experte, der Paracelsus als Universalgelehrten ehrte. Die medizinischen Schriften des Hohenheimers hätten, so Dopsch, jedoch in kaum einem Bereich eine Innovation dargestellt, sondern wären eher als Abbild des zeitgenössischen stateof-the-art anzusehen und kaum nachhaltig und langfristig rezipiert worden. Lediglich die heroischen Quecksilberkuren zur Therapie der Syphilis wurden bis ans Ende des 19. Jahrhun-

1 Robert JÜTTE, Geschichte der Alternativen Medizin. Von der Volksmedizin zu den unkonventionellen Therapien von heute (München 1996), 9. 
derts angewandt, jedoch nicht als naturheilkundliche, alchimistisch-inspirierte Herangehensweise alternativer Therapeutik, sondern als fester Bestandteil des Methodenrepertoires der universitär gelehrten Medizin.

In ihrem zeitlich nahen, ebenfalls an der Schwelle zur Neuzeit angesiedelten Beitrag untersuchte Nina Daniela MAIER (Salzburg/Mallorca) die Wahrnehmung und Behandlung psychischer Erkrankungen. Anhand unterschiedlicher Quellengattungen (Pathographien, Rezepten) zeigte die Referentin das Repertoire möglicher therapeutischer Interventionen im Hinblick auf die Heilung oder Besserung psychisch Kranker auf. Auch Maier wies auf die Problematik der Begrifflichkeiten hin, denn eine Definition dessen, was in Spätmittelalter und Früher Neuzeit als alternativ, mit anderen Worten entgegen der etablierten medizinischen Norm empfunden worden sei, wäre sowohl geographischen wie sozialen Unterschieden unterworfen gewesen. Zudem machte der Fokus auf die PatientInnen deutlich, dass oftmals neben der etablierten Humoralpathologie naturkundliche, diätetische und magische Elemente miteinbezogen wurden und somit von einem Neben- und Miteinander bzw. von einer ,intensiven Symbiose“ unterschiedlicher therapeutischer Ansätze auszugehen ist.

\section{Religiös-magische Medizin}

Mit insgesamt vier Beiträgen aus unterschiedlichen Jahrhunderten war der Themenbereich der religiös-magischen Medizin vergleichsweise gut vertreten. Anhand von Quellen aus dem 18. Jahrhundert thematisierte Gerhard AMMERER (Salzburg) im ersten Teil seines Vortrags Teufelsaustreibungen als alternative Methode der Behandlung von Kranken, bei denen ein dämonischer Ursprung des Leidens angenommen wurde. In einem zweiten Teil veranschaulichte Ammerer am Beispiel Johann Joseph Gaßners (1727-1779), welcher als Exorzist und Wunderheiler agierte und enormen Zuspruch aus der Bevölkerung erfuhr, wie übergeordnete Interessen - in seinem Fall jene der geistlichen und weltlichen Autoritäten, welche die durch Gaßner neu aufkeimende, übersteigerte Teufelsfurcht kritisierten und die Volksaufklärung gefährdet sahen - die Karriere eines Heilanbieters jäh beenden oder forcieren konnten. Tatsächlich ging ein anderer alternativer Heilanbieter - Franz Anton Mesmer (1734-1815) -, dessen animalischer Magnetismus große Ähnlichkeit zu Gaßners Praktiken zeigte, als Gewinner aus der Kontroverse rund um Gaßners Wunderheilungen hervor.

Ehler VOSS (Siegen) wählte eben jenen Konflikt als Ausgangspunkt seiner Erläuterungen zur „Immanentisierung spiritistischer Wirkungen im 19. Jahrhundert und ihre Auswirkungen auf die Konzeption von Heilung". Voss sieht demnach den Ursprung der von ihm untersuchten „Kultur des medialen Heilens“ im europäischen Spiritismus des 19. Jahrhunderts begründet. Adaptionen und Weiterentwicklungen des animalischen Magnetismus wurden dabei ebenso skizziert wie die verschiedenen modernen Techniken und Methoden des medialen Heilens (Reiki, Schamanismus, Familienaufstellung nach Hellinger, Lehre Bruno Grönings vom göttlichen Heilstrom). In der Praxis verdichten sich, so Voss, meist mehrere Methoden und Praktiken zu der bereits erwähnten „Kultur des medialen Heilens“.

Alexander FRIEDMAN (Heidelberg) beschäftigte sich schließlich mit einem rezenten Phänomen medialen Heilens, nämlich den Fernhypnosen des ukrainischen Psychiaters Anatolij Kašpirovskij (*1939). Dessen vermeintlich heilende Kräfte, die er in eigenen Fernsehshows vor Publikum anwandte, erzeugten ab dem Ende der 1980er Jahre großes mediales Echo über 
den (post-)sowjetischen Raum hinaus. Das Spektrum der durch den Wunderheiler behandelten körperlichen Gebrechen reichte von kindlichem Bettnässen über diverse organische Erkrankungen bis hin zu Diabetes und Unfruchtbarkeit. Die wechselhafte Karriere des stark polarisierenden Kašpirovskij war und ist insbesondere durch seine ambivalente Verbindung zur russischen Politik gekennzeichnet, Verbindungen zum KGB werden ihm bis heute nachgesagt.

Indem sie sich der Pastoralmedizin und ihrer Nähe zu alternativen medizinischen Konzepten wie etwa der Homöopathie widmete, siedelte Maria HEIDEGGER (Innsbruck) ihren Beitrag im Spannungsfeld zwischen religiöser und biodynamischer Medizin an. Mit ihrem exemplarischen, äußerst quellennahen Zugang machte sie die Affinität vieler Geistlicher zur Medizin, insbesondere aber zur Homöopathie deutlich. Die Ausführungen im vorliegenden Band fokussieren die Zeit um die Mitte des 19. Jahrhunderts und machen die verschiedenen beteiligten Positionen deutlich: die homöopathisch tätigen Geistlichen und ihre Motive werden dabei ebenso wie die Nachfrageseite in der meist ländlichen Bevölkerung, die Bedenken und Konkurrenzängste der medizinischen Professionisten sowie die (oft vergeblich) regulierenden Bestrebungen der Obrigkeiten portraitiert.

\section{Naturheilkunde und Lebensreformbewegung}

Neben der Homöopathie, die auch im aktuellen medizinischen Diskurs eine Rolle spielt, wurden im Verlauf der Tagung auch weniger bekannte alternative Konzepte diskutiert. Die folgenden Beiträge sind allesamt im Lichte der Lebensreform-Bewegung des 19. Jahrhunderts zu sehen und stellten einen weiteren Schwerpunkt der Tagung dar. Christina VANJAs (Kassel) Vortrag über „Gesunde Kochbücher - Diätetik in Kuranstalten um 1900“ erlaubte Einblicke in die Welt der Speisesäle von Sanatorien der Jahrhundertwende (Bircher-Benners „Lebendige Kraft“, Kelloggs „Battle Creek Sanitarium“, Lahmanns „Weißer Hirsch“ oder die Dresdener Kurhäuser des Friedrich Eduard Bilz). Ob es nun darum ging, die physische oder psychische Konstitution durch Mastkuren zu stärken oder den Körper durch spezielle Fastenkuren von schädlichen Stoffen zu befreien, war all diesen Reformbewegungen gemein, dass man mit der Publikation von Kochbüchern nicht nur eine Fortsetzung der Kuren zuhause ermöglichen, sondern die propagierte Diätetik einem breiteren Publikum zugänglich machen wollte. Damit beeinflusste man ganz maßgeblich das Essverhalten bis in die Gegenwart. Denn was heute teils als moderner Trend oder innovativer Ansatz gefeiert wird, fand seinen Ursprung häufig in der Diätetik der Sanatorien des beginnenden 20. Jahrhunderts, deren Ideen wiederum meist auf die antike Diätetik (sex res non naturales) rekurrierten.

In Sabine VEITS-FALKs (Salzburg) Vortrag über „Alternative Heilmethoden als Berufsfeld der ersten Ärztinnen der Habsburgermonarchie“ wurde neben einem Überblick über die Biographien zweier um 1900 naturheilkundlich tätiger „Schweizerärztinnen“ auch deren äußerst erfolgreiche publizistische Tätigkeit im Bereich der Ratgeberliteratur für ein vornehmlich weibliches Publikum angesprochen. Die Bemühungen Anna Fischer-Dückelmanns (18561917) sowie Gisela Lucci-Purtschers (1871-1959) im alternativ-medizinischen Feld der Naturheilkunde Fuß zu fassen, sind dabei wohl als strategische Wahl zu erkennen, denn die wenigen Medizinerinnen der Habsburgermonarchie versuchten, sich in fachlicher Hinsicht häufig in ,jungen“ Fachrichtungen oder Nischenfächern zu etablieren. In den skizzierten Biographien gipfelte das Engagement für die Naturheilkunde in beiden Fällen in der Eröffnung 
eigener Kuranstalten oder Praxen. Bei Fischer-Dückelmann ist die Eröffnung einer naturheilkundlichen Praxis in Dresden-Loschwitz wohl als Strategie zu sehen, denn über die Kurfreiheit konnte sie ihren Beruf auch außerhalb der Schweiz ausüben. Bezeichnenderweise siedelten sich die naturheilkundlich tätigen Ärztinnen häufig im Umfeld bereits etablierter Naturheilanstalten, etwa in Dresden oder Zürich, an.

Irmtraut SAHMLAND (Marburg) thematisierte eine weitere Facette der Lebensreform-Bewegung des 19. Jahrhunderts und stellte die Entwicklung einer Kleidungshygiene in Form des so genannten „Woll-Regimes“ durch Gustav Jäger (1832-1917) ins Zentrum ihrer Ausführungen. Jägers dualistisches System, das auf so genannten Lust- und Unluststoffen basierte, war dabei allerdings nicht nur auf das Tragen von gesunden Textilien, der so genannten Normalkleidung, beschränkt, sondern griff etwa mit der „Desodorierung“ (Lüften) oder dem Schwitzen durch körperliche Betätigung den Kanon antiker Diätetik auf. Jäger versuchte zudem die Nützlichkeit seiner Reformkleidung anhand naturwissenschaftlicher Messung theoretisch zu fundieren.

Mit einem besonderen Aspekt des naturheilkundlichen Feldes beschäftigte sich Florian MILDENBERGER (Frankfurt/Oder) in seinem Vortrag über „Komplementäre Heilweisen und das Sexualleben - ein diffiziles Verhältnis 1880-1930“. Was sich zunächst als ein grundsätzlich positives Verhältnis konstituierte und mit großem Zuspruch von der Nachfrageseite begann, änderte sich mit der Debatte um die bestmögliche Therapie der Syphilis schlagartig. Die Ablehnung der Salvarsantherapie und die gleichzeitige Propagierung eines abstinenten Lebenswandels entpuppten sich als Sargnagel für die Naturheilverfahren im Bereich der sexuellen Therapien und führten zu einer Exklusion aus dem Sexualdiskurs. Abschließend stellte Mildenberger dar, wie diese Kontroverse den Weg der unterschiedlichen „Heilpraktiker“ in den Nationalsozialismus ebnete.

\section{Akteure einer alternativen Medizin}

Bettina NOAK (Berlin) präsentierte in ihrem Beitrag die niederländischen Ärzte Cornelis Bontekoe (1647-1685) und Steven Blankaart (1650-1702) und ihre Auseinandersetzungen mit der „Schulmedizin“ des 17. Jahrhunderts. Die niederländischen Ärzte, welche sich in ihren zahlreichen Schriften vehement gegen die gelehrten Traditionen (Humoralpathologie) auflehnten, etablierten eine cartesianisch-inspirierte Lehre der Medizin, welche auf der naturwissenschaftlich geleiteten Idee des Blutkreislaufes basierte. Ihre rationalen, anthropologischen Ansätze, die auch eine spezielle Form der Diätetik inkludierten, versuchten Bontekoe und Blankaart durch die beständige Publikation allgemeinverständlicher, volkssprachlicher Schriften zu popularisieren. Insbesondere Cornelis Bontekoe agierte jenseits der universitär etablierten Medizin seiner Zeit und hätte sich, so Noak, wohl selbst als Alternativmediziner bezeichnet, wobei aus dem Beitrag jedoch nicht klar wird, in wie weit die genannten Gelehrten auch tatsächlich praktizierten.

In seinem Beitrag „An den Rand gedrängt? Die Praxis des Thurgauer Laienheilers Gottfried Wachter (1776-1861)“ wählte Alois UNTERKIRCHER (Zürich) hingegen einen dezidiert praktischen Zugang. Sehr anschaulich erläuterte Unterkircher, wie im Zuge der Neuorganisation des kantonalen Sanitätswesens am Ende des 18. Jahrhunderts einem etablierten, jedoch universitär nicht approbierten Heiler der Stempel des „Laienheilers“ und somit aus 
heutiger Sicht Vertreters einer alternativen Heilmethode aufgedrückt wurde. Dass diese Kategorisierung nur auf den ersten Blick standzuhalten vermag, wurde anhand Unterkirchers Untersuchung des Therapieangebots deutlich, denn dies unterschied sich kaum von dem eines Wundarztes oder akademischen Arztes seiner Zeit. In Wachters Fall war es wohl in erster Linie die fehlende formale Approbation, die ihn offiziell als Laienheiler und damit alternativen Heilanbieter klassifizierte.

Alexandra LEWENDOSKI (Berlin) beschäftigte sich in ihrem Beitrag mit dem ganzheitlichen Konzept der Osteopathie, insbesondere mit deren Begründer, dem US-Amerikaner Andrew Taylor Still (1828-1917). Still stellte die Osteopathie nicht als eine alternative oder komplementäre Heilweise vor, sondern betrachtete seine Lehre als ein naturwissenschaftlich und naturphilosophisch begründetes, kohärentes Heilsystem, die Lewendoski mit den Theorien Gottfried Wilhelm Leibniz vergleicht. Beeinflusst von den technischen Errungenschaften seiner Zeit, sah Still den menschlichen Körper als „,bedeutendste aller Maschinen“, die Körper-Maschine-Metapher wird somit zu einem zentralen Erklärungsansatz. Zudem zeigte sich Still der religiösen Tradition der Methodisten verpflichtet und bezeichnete seine Lehre als Gesetz Gottes.

\section{Aktuelle Trends}

Einen Überblick über aktuelle Trends am komplementärmedizinischen Markt boten der Mediziner Peter WEILER (Salzburg) sowie die Medizinanthropologin Andrea PAMMER (Wiener Neustadt). Weiler zeigte das Spektrum der derzeit von der Ärztekammer (ÖÄK) anerkannten komplementären Methoden in Österreich auf. Neben approbierten Ärzten, die alternative Methoden wie etwa die Akupunktur, Homöopathie oder die Anthroposophische Medizin nach Erlangung eines offiziellen ÖÄK-Diploms legal ausübten, gebe es, laut Weiler, einen großen „therapeutischen Graubereich“, in welchem andere Berufsgruppen diverse alternativ- und komplementärmedizinische Dienstleistungen anbieten. Weiler betonte, dass in Zukunft die individualisierte Medizin im Sinne einer Verschränkung von Schul- und Komplementärmedizin immer mehr an Bedeutung gewinnen werde. Andrea Pammer thematisierte den aktuell vorherrschenden medizinischen Pluralismus aus theoretischer Perspektive und präsentierte ihre Forschungen zum Medizinpluralismus in Österreich anhand der Fokussierung auf die asiatischen Heilmethoden (Traditionelle Chinesische Medizin, Ayurveda, Tibetische Medizin). In ihrem Vortrag wurde erneut auf die Dominanz der „Biomedizin“ über die alternativen Methoden hingewiesen sowie der Wirtschaftsfaktor asiatische Medizin analysiert. Auch aktuell spielen, so Pammer, ökonomische und politische Interessen in der Verbreitung alternativ- und komplementärmedizinischer Methoden eine gewichtige Rolle.

Im Verlauf der Tagung kristallisierten sich etliche Gemeinsamkeiten und Überschneidungen in den einzelnen Beiträgen heraus. Dabei bildeten sich gewisse thematische Schwerpunkte, welche im Folgenden näher beleuchtet werden sollen. 


\section{Geburt alternativer Ansätze}

Die Evolution alternativer Ideen wurde in mehreren Vorträgen thematisiert und analysiert. Dabei zeichneten sich zwei Entwicklungslinien ab, die in der Praxis aber durchaus miteinander verschmelzen konnten. Während nämlich vielfach eine grundlegende Unzufriedenheit mit dem herrschenden medikalen System zur Entfremdung von der „Schulmedizin“ und so zur Generierung alternativer Konzepte führte (z. B. Paracelsus, Bontekoe), scheint die Krise des Individuums als Auslöser und Wegbereiter alternativer Ansätze ein ebenso häufig wiederkehrendes Motiv gewesen zu sein. So führte etwa die Unzufriedenheit mit der eigenen physischen Verfassung, gepaart mit einer grundsätzlichen Kritik an den Methoden der universitär gelehrten Medizin, dazu, dass Gustav Jäger seinen Lebenswandel überdachte und seine Idee des Wollregimes entwickelte. Dabei war nicht nur seine kränkliche Konstitution ausschlaggebend gewesen, sondern auch die Tatsache, dass Jäger eine Vorlesung für Anthropologie halten sollte. Deren Inhalte zur Gesundheitslehre wollte er nicht nur theoretisch fundiert wissen, sondern praktisch vorleben. Dabei sei er sich aber vorgekommen, „,wie ein Kahlköpfiger, der Haarbalsam anpreist", um wie Sahmland bei Jägers Metapher zu bleiben. Christina Vanja nannte im Zusammenhang mit der Hinwendung der Brüder Just zur Naturheilkunde ebenfalls die Krankheit Adolf Justs als auslösendes Moment. Ehler Voss thematisierte in seinem Beitrag die Rekrutierung von spirituellen Medien aus einer tiefgreifenden persönlichen Krise heraus. Und auch bei Andrew Taylor Still, dem Begründer der Osteopathie, wurde der kreative Prozess zur Erschaffung eines neuen, ganzheitlichen medizinischen Konzeptes erst durch den Meningitis-Tod mehrerer naher Angehöriger in Gang gesetzt. Ob eine persönliche Krise dazu führte, dass Cornelis Bontekoe sich am Ende des 17. Jahrhunderts aufs Land zurückzog und sich, laut Noaks Beitrag, dem Studium der Schriften Descartes und antiker Autoren wie Hippokrates und Demokrit widmete, ist nicht überliefert, doch zeigt sich in seiner Person ein weiteres Element ,alternativer“ Ideenentwicklung: die Nutzbarmachung und Weiterentwicklung bestehender Theorien sowie die Rückbesinnung auf die Prinzipien der antiken Diätetik.

\section{Antike Diätetik}

Im Zentrum alternativer Konzepte steht vielfach das Postulat einer gesunden Lebensweise, welche von den diversen Heilanbietern unterschiedlich definiert und interpretiert wird. Im Rahmen der Tagung wurde die Rückbesinnung auf die antike Diätetik zur augenscheinlichen Parallele in etlichen Beiträgen. Einen gelungenen Einstieg in die Thematik lieferte Christina Vanja in ihrem unterhaltsamen Abendvortrag, welcher die Diätetik der Kuranstalten um 1900 auf die Tradition der „sex res non naturales“ (Galen) zurückführte. Neben frischer Luft, körperlicher Betätigung, ausreichend Schlaf, einem gesunden Stoffwechsel und einer ausgeglichenen psychischen Verfasstheit sei ein besonderes Augenmerk auf eine gute Ernährung zu legen. Diese Devise fand - wie Vanja anhand literarischer Zeugnisse und diverser Kochrezepte eindrucksvoll belegen konnte - in den Sanatorien der Jahrhundertwende in unterschiedlichen Ausprägungen Anwendung. Während die Lebensreformbewegung des 19. Jahrhunderts das antike Wissen rund um die Diätetik als neue Errungenschaft zelebrierte, zeigte etwa der Beitrag Maiers, dass diätetische Maßregeln unterschiedlichster Ausprägung bereits in der Frühen Neuzeit fest im medikalen Wissen der Zeit verankert waren. So konstatierte Maier: „In die 
Heilkraft der Diätetik vertrauten alle untersuchten Fallbeispiele. Vor allem aus den adeligen Schichten sind zahlreiche Ernährungs- und Bewegungspläne überliefert". In der exemplifizierend herangezogenen Pathographie des Gelehrten Hieronymus Wolf (1516-1580) finden sich ebenfalls Hinweise auf einen nicht näher definierten Ernährungsplan sowie auf die Berücksichtigung ,positiver Luft- und Raumverhältnisse“. Auch Bettina Noak thematisierte die Diätetik im Werk Cornelis Bontekoes und Steven Blankaarts. Beide definierten eine allen Krankheiten zugrundeliegende Ursache, die in den Schärfen der Körpersäfte liege. Bei Bontekoe war die Ursache der Skorbut, der scharfe und somit krankmachende Stoffe entstehen ließ. Bontekoe riet zur Vermeidung ebendieser Prozesse zum ausgiebigen Kaffee- und Teekonsum, sah allerdings auch die Seelenruhe als Quell der Lebenskraft und Mittel zur Gesunderhaltung. Gustav Jäger stellte, laut Irmtraut Sahmland, ein spezifisches „Selbstgift“ in das Zentrum seines „Systems“. Der Entstehung dieses systemimmanenten Giftes sei durch die beständige Entfettung und Entwässerung des Körpers entgegenzuwirken. Jäger empfahl neben dem Tragen der durch ihn konzipierten Wollkleidung überdies körperliche Ertüchtigung, Schwitzkuren sowie die Rücksichtnahme auf gute Ventilation und Belüftung. Am Beispiel Anatolij Kašpirovskijs lässt sich die Diätetik ebenfalls thematisieren, denn der Wunderheiler trat als Verfechter einer gesunden Lebensweise auf und präsentierte sich selbst als durchtrainiert und körperlich fit. Laut Kašpirovskij würden vier Faktoren die Gesunderhaltung des Körpers begünstigen: dabei berief sich der studierte Mediziner auf die Gene, sportliche Betätigung, die Abstinenz bei Genussmitteln wie Alkohol und Nikotin sowie auf die „chronische Verliebtheit“, welche wohl als positive Grundeinstellung interpretierbar ist.

\section{Heilerisches Selbstverständnis}

Ein weiterer Schwerpunkt war der Rekonstruktion des Selbstverständnisses der Alternativmediziner gewidmet. Natürlich lag und liegt es in der Natur der Sache, dass die Verfechter einer alternativen Heilweise ihre Theorien so weit als möglich zu popularisieren gedachten und dies mit der notwendigen Vehemenz in die Tat umsetzten. Am Beispiel der Tagungsvorträge bzw. der in diesem Band verschriftlichten Beiträge kann das Spektrum der Abgrenzungstechniken sowie der Legitimierung des eigenen Handelns aufgezeigt werden. Eine akteurszentrierte Sichtweise kam etwa in Bettina Noaks Beitrag zum Cartesianer Cornelis Bontekoe zum Tragen, indem sie ihn als „Vorkämpfer der Wahrheit, dessen Gegner nichts als Lüge und Gewinn im Sinne" hätten, portraitierte. Ihre Einschätzung der Persönlichkeit Bontekoes unterstrich Noak durch den von Bontekoe selbst gewählten Verweis auf den antiken Diana-Kult als eine Metapher auf seine eigene Rolle des „Apostels der neuen, heilsbringenden Lehre“. So stellte sich Bontekoe, aber auch sein weniger polemisierender Zeitgenosse Steven Blankaart, in seinen Schriften vehement gegen die etablierte Galenische Medizin. Den beiden Cartesianern lassen sich volkserzieherische Tendenzen zuschreiben, denn die Publikation ihrer volkssprachlichen Texte popularisierte nicht nur ihre Lehren, sondern war wohl auch geeignet, die Emanzipation des Kranken voranzutreiben. Eine Emanzipation von den Methoden und Theorien der zeitgenössischen Medizin des 19. Jahrhunderts stellte ferner der Kleiderhygieniker Gustav Jäger ins Zentrum seines „Systems“. So basierte seine Kritik an der Medizin darauf, dass sich diese lediglich mit Sekundärphänomenen beschäftige und das Wesen der zugrundeliegenden Krankheit nicht erkenne. Sein Wollregime sei „deutlich überlegen“, zwar kein Allheilmittel, 
jedoch in etwa $90 \%$ der Fälle als Prophylaxe äußerst erfolgreich, um der, laut Jägers Theorie, ursächlich krankheitsauslösenden Selbstvergiftung vorzubeugen und sich so unabhängig von der Deutungsmacht der Schulmedizin zu machen. Ein starkes heilerisches Selbstverständnis ist auch Andrew Taylor Still nicht abzusprechen, bezeichnete er sich schließlich selbstbewusst als „Vater der Osteopathie“. Der Erfolg seines geistigen Kindes sei in Gott gegründet und daher von oberster Instanz legitimiert.

Während sich die genannten Persönlichkeiten lediglich als Propheten einer neuen medizinischen Lehre, eines neuen Lebensstils sahen, der ohne die direkte Präsenz der Heilerpersönlichkeiten umsetzbar und wirksam war, bildeten andere Heiler selbst das Zentrum ihrer Lehre. Ehler Voss nannte in diesem Zusammenhang das Phänomen Bruno Gröning, welcher nach dem Zweiten Weltkrieg als Geistheiler in Deutschland auftrat. Die durch ihn strömende göttliche Kraft kanalisierte Gröning, nach eigenen Aussagen, im so genannten Heilstrom. Doch nicht nur der persönliche Kontakt zum Medium, sondern auch die Teilnahme an Veranstaltungen und die Verwendung so genannter Gröningkugeln (aus Stanniolfolie), welche Haare, Nägel oder Blutstropfen Grönings enthielten und somit den wertvollen Heilstrom weitergaben, konnte seiner Anhängerschaft Linderung bei verschiedenen Leiden verschaffen. Auch der ukrainische Wunderheiler Anatolij Kašpirovskij stellte sich selbst in den Mittelpunkt seiner Heilversuche. Neben seinen Fernsehauftritten, bei denen er per Fernhypnose die Zuseher zu heilen versuchte, sollte die intensive Betrachtung seines Portraits ebenfalls zu den gewünschten Heilerfolgen führen.

Lässt sich das Selbstverständnis und die Überzeugung für ihre Lehren bei den vorgenannten Alternativmedizinern anhand ihrer zahlreichen Schriften oder dem erfolgten medialen Interesse nachvollziehen, so wird das gelebte Selbstbewusstsein anderer Heilerpersönlichkeiten nur indirekt fassbar. Nicht immer musste die Opposition laut artikuliert werden, vielfach reichte die bewusste Nichtbefolgung obrigkeitlicher Verordnungen als ,alternativer“ Akt. So konnte Alois Unterkircher anhand der statistischen Auswertung der Krankenjournale des Thurgauer Laienheilers Gottfried Wachter (1776-1861) zeigen, dass, obwohl diesem von der Medizinalverwaltung lediglich die Ausübung einer eingeschränkten chirurgischen Praxis erlaubt worden war, Wachter nichtsdestotrotz in mehr als der Hälfte aller Fälle innere Kuren bei seinen PatientInnen anwandte. Während Wachter trotz des Mangels an formaler Approbation quasi dennoch die Funktion und das Repertoire eines akademischen Arztes übernahm, bot der Geistliche Johann Joseph Gaßner seinen PatientInnen tatsächlich einen alternativen Ansatz. Gaßner hatte sich den Ruf eines Wunderheilers erarbeitet und konnte auf eine ansehnliche Anhängerschaft verweisen. Nicht weniger als 20.000 Kranke soll er beispielsweise in wenigen Monaten durch seine spezifische Form des Exorzismus von ihren Leiden befreit haben. Trotz Strafversetzung und mehrerer Verweise praktizierte Gaßner bis zu seinem Tod zum Wohle der auf ihn und seine Teufelsaustreibungen vertrauenden Mitmenschen. Auch die homöopathisch tätigen Geistlichen aus Maria Heideggers Beitrag übten Ungehorsam gegen die kirchlichen Obrigkeiten und setzten ihre heilerischen Tätigkeiten trotz Verboten und dienstrechtlichen Konsequenzen munter fort. Dabei bemühten sie das vielzitierte Gesetz der christlichen Nächstenliebe, welches ihnen untersagte, notleidenden Personen ihre therapeutische Hilfe zu verwehren. Der Aspekt der Popularisierung lässt auf das Selbstbewusstsein der Alternativmediziner schließen. Einen Hinweis darauf erhalten wir aus Gerhard Ammerers Beitrag, indem er erwähnt, dass Gaßner mit seinen Exorzismen gerne vor Publikum aufgetreten sei. Beinahe absurde Ausmaße nimmt die Medienwirksamkeit alternativer Methoden im Beitrag von Alexander Friedman an. 
Nicht weniger als zehn Millionen Menschen soll der ukrainische Psychiater Anatolij Kašpirovskij, nach eigenen Angaben, durch seine Methode der Fernhypnose über das russische Staatsfernsehen in den 1980er und 1990er Jahren kuriert haben. Zum Medienstar gehypt und erfolgreich vermarktet, regte sich aber bald Kritik an der übersteigerten Persönlichkeit des Psychiaters und seinen zweifelhaften Methoden. Insbesondere Mediziner und die orthodoxe Kirche traten offen gegen Kašpirovskij auf. Dessen Ego schien daran jedoch keinen Schaden zu nehmen, er strotzte regelrecht vor Selbstbewusstsein, engagierte sich u. a. auch in der Politik und trat, eigenen Angaben zufolge sehr erfolgreich, als Vermittler in einem Geiseldrama auf. Sein Vertrauen in die eigenen Fähigkeiten gipfelte sogar in konkreten Drohungen gegen Leib und Leben einiger Kriminalbeamter, die gegen den „neuen russischen Rasputin“, dem die Ausübung seiner Heilertätigkeit unter Boris Jelzin untersagt wurde, ermittelten.

Es ließen sich durchaus noch weitere Parallelen in den einzelnen Beiträgen skizzieren, Vergleiche anstellen und Perspektivenwechsel vollziehen, doch um nicht repetitiv zu werden, sei an dieser Stelle lediglich auf das durchaus ambivalente Verhältnis der Alternativmedizin zur Politik verwiesen, welches etwa bei Mildenberger oder Friedman ausführlich diskutiert wird. Insgesamt zeigten die unterschiedlichen Zugänge und historischen Beispiele die Vielfalt des medizinischen bzw. therapeutischen Angebots abseits der universitär etablierten und approbierten medizinischen Wissenschaft auf. Die diesjährige Tagung des Vereins für Sozialgeschichte der Medizin übte aber auch einmal mehr Kritik am Paradigma der „Medikalisierung“. Dabei wurde, im Sinne des Tagungstitels über Alternativen für das Gegensatzpaar der „Alternativ- vs. Schulmedizin" nachgedacht und resümierend für eine kritische Verwendung der vorherrschenden Terminologie plädiert.

\section{Informationen zur Autorin}

Marina Hilber, Mag. Dr., wissenschaftliche Projektmitarbeiterin am Institut für Geschichtswissenschaften und Europäische Ethnologie der Universität Innsbruck, derzeit in Karenz

Forschungsschwerpunkte: Geschichte der Gebärhäuser, Professionalisierungsgeschichte der Hebammen, Sozialgeschichte des frühneuzeitlichen Tiroler Bergbaus 